\title{
Land scale biogeography of arsenic biotransformation genes in estuarine wetland
}

\author{
Si-Yu Zhang, ${ }^{1,2}$ Jian-Qiang Su, ${ }^{3}$ Guo-Xin Sun, ${ }^{1}$ \\ Yunfeng Yang, ${ }^{4 *}$ Yi Zhao, ${ }^{3}$ Junjun Ding, ${ }^{5}$ \\ Yong-Shan Chen, ${ }^{3}$ Yu Shen, ${ }^{3}$ Guibing Zhu, ${ }^{6}$ \\ Christopher Rensing ${ }^{3,7}$ and Yong-Guan Zhu ${ }^{1,3 * *}$ \\ ${ }^{1}$ State Key Lab of Urban and Regional Ecology, \\ Research Center for Eco-Environmental Sciences, \\ Chinese Academy of Sciences, Beijing, 100085, China. \\ ${ }^{2}$ University of Chinese Academy of Sciences, Beijing, \\ 100049, China. \\ ${ }^{3}$ Key Laboratory of Urban Environment and Health, \\ Institute of Urban Environment, Chinese Academy of \\ Sciences, Xiamen, 361021, China. \\ ${ }^{4}$ State Key Joint Laboratory of Environment Simulation \\ and Pollution Control, School of Environment, Tsinghua \\ University, Beijing, 100084, China. \\ ${ }^{5}$ Key Laboratory of Dryland Agriculture, Ministry of \\ Agriculture, Institute of Environment and Sustainable \\ Development in Agriculture, Chinese Academy of \\ Agricultural Sciences, Beijing, 100081, China. \\ ${ }^{6}$ Key Laboratory of Drinking Water Science and \\ Technology, Research Center for Eco-Environmental \\ Sciences, Chinese Academy of Sciences, Beijing, \\ 100085, China. \\ ${ }^{7}$ Fujian Provincial Key Laboratory of Soil Environmental \\ Health and Regulation, College of Resources and the \\ Environment, Fujian Agriculture \& Forestry University, \\ Fuzhou, China.
}

\section{Summary}

As an analogue of phosphorus, arsenic (As) has a biogeochemical cycle coupled closely with other key elements on the Earth, such as iron, sulfate and phosphate. It has been documented that microbial genes associated with As biotransformation are widely present in As-rich environments. Nonetheless, their presence in natural environment with low As levels remains unclear. To address this issue, we investigated the abundance levels and diversities of

Received 16 January, 2017; revised 2 April, 2017; accepted 18 April, 2017. For correspondence. *E-mail yangyf @tsinghua.edu.cn; Tel. +86 10 62784692; Fax: +86 10 62794006. **E-mail ygzhu@ rcees.ac.cn; Tel. +86 10 62936940; Fax +86 1062923563 .
aioA, arrA, ars $C$ and ars $M$ genes in estuarine sediments at low As levels across Southeastern China to uncover biogeographic patterns at a large spatial scale. Unexpectedly, genes involved in As biotransformation were characterized by high abundance and diversity. The functional microbial communities showed a significant decrease in similarity along the geographic distance, with higher turnover rates than taxonomic microbial communities based on the similarities of 16S rRNA genes. Further investigation with niche-based models showed that deterministic processes played primary roles in shaping both functional and taxonomic microbial communities. Temperature, $\mathrm{pH}$, total nitrogen concentration, carbon/nitrogen ratio and ferric iron concentration rather than As content in these sediments were significantly linked to functional microbial communities, while sediment temperature and $\mathrm{pH}$ were linked to taxonomic microbial communities. We proposed several possible mechanisms to explain these results.

\section{Introduction}

Arsenic (As) is a toxic and carcinogenic element that is widely distributed in the environment. Microbe-mediated As biotransformation is an ancient mechanism that emerged at least 2.72 billion years ago (Lebrun et al., 2003; Duval et al., 2008; Sforna et al., 2014) and significantly modulate the fate of As in the environment via As respiratory oxidation and reduction, arsenate $(\mathrm{As}(\mathrm{V}))$ cytoplasmic reduction and arsenite (As(III)) methylation (Zhu et al., 2014). As(III) oxidase (Aio) catalyses the respiratory oxidation of $\mathrm{As}$ (III) by either detoxifying it to less toxic $\mathrm{As}(\mathrm{V})$, or coupling it to ATP production (Slyemi and Bonnefoy, 2012). Aio's large subunit (AioA) has been well characterized in both bacteria and archaea (Lebrun et al., 2003). As(V) respiratory reduction in bacteria couples $\mathrm{As}(\mathrm{V})$ reduction to anaerobic heterotrophic growth (Ahmann, 1994; Bhattacharjee and Rosen, 2007), for which arrA gene encoding the large catalytic subunit (ArrA) serves as a reliable marker (Malasarn et al., 2004). Cytoplasmic $\mathrm{As}(\mathrm{V})$ reduction is a detoxification mechanism followed by $A s(I I I)$ efflux, and it is catalysed by $A s(V)$ reductase (ArsC) (Mukhopadhyay et al., 2002; Silver and 
Phung, 2005). As(III) methylation catalysed by As(III) $S$ adenosylmethionine methyltransferase (ArsM) is an alternative As detoxification mechanism that methylates inorganic As(III) to less toxic organic As and volatile As such as mono-, di- and trimethylarsines (Qin et al., 2006; Yin et al., 2011b; Wang et al., 2014; Zhu et al., 2014).

Microbial genes involved in arsenate $(\mathrm{As}(\mathrm{V}))$ respiratory reduction $(\operatorname{arr} A), A s(\mathrm{~V})$ detoxification reduction (arsC), arsenite (As(III)) oxidation (aioA) and As(III) methylation (arsM) are detected with high abundance and diversity in As-rich environments such as paddy soils (Zhang et al., 2015), mine fields (Luo et al., 2014) and spring or brine sediments (Escudero et al., 2013), demonstrating the presence of microbes capable of As biotransformation in these environments. The underlying mechanisms of these microbial biogeographic patterns can be classified into two types: deterministic (also termed niche-based) processes, and stochastic or neutral processes (Martiny et al., 2006). The deterministic processes imposed by environmental filters select certain species according to their environmental preferences (Aguilar et al., 2014; Hambright et al., 2015), while stochastic processes are driven by ecological drift and dispersal limitation (Martiny et al., 2011; Zhou et al., 2014). Notably, most of the current biogeographic microbial studies are focussed on 16S rRNA genes, despite a resurging interest in understanding the microbial biogeographic patterns of functional genes (Green et al., 2008), which serve as the basis of the biotransformation of various elements on the earth and contribute to the elemental cycle.

Arsenic biotransformation has been detected in natural environments with low As levels (Zhang et al., 2017). In estuarine and coastal wetlands, at interface between terrestrial and coastal ecosystems (Zedler and Kercher, 2005), the activity of As(V)-reducing microbes is thought to be the key driver for As release from sediments to aquifers (Islam et al., 2004), while As(III)-oxidizing microbes are able to decrease As mobility and bioavailability (Bhattacharya et al., 2007). Together, these two microbial groups determine the dynamic release of inorganic As species from estuarine sediments to costal aquifers. As(III) methylation has been suggested as a link between surface and subsurface As cycling (Maguffin et al., 2015), and the volatile As species generated during this process can further facilitate As emissions to the atmosphere (Chilvers and Peterson, 1987; Mestrot et al., 2011). Therefore, it is of great interest to investigate the presence and geographic distribution patterns of microbes involved in As biotransformation in estuarine sediments.

Here, we examined the microbial biogeography associated with As biotransformation in 70 samples collected from 14 estuarine sediments in Southeastern China. To our knowledge, this is the first study of microbial biogeography associated with As biotransformation in natural environments, which typically with low As levels that we encounter today (Kulp, 2014). Quantitative PCR (qPCR) methods and terminal restriction fragment length polymorphism (T-RFLP) analysis were used to investigate the abundance levels and geographic distribution patterns of aio $A$, arrA, ars $C$ and ars $M$ genes respectively. Highthroughput MiSeq sequencing was performed to profile the taxonomic microbial community via the 16S rRNA genes and the functional microbial community via the arsM genes, owing to its important role in linking surface and subsurface arsenic cycling (Maguffin et al., 2015), and the limited knowledge of the ars $M$ genes in the environment since it was amplified only from the paddy soils three years ago (Jia et al., 2013). We aim to address the following questions: (i) Are microbial genes involved in As biotransformation widely present in the estuarine wetlands with low As levels? (ii) Do As biotransformation genes have biogeographic patterns? (iii) Which process drive the biogeographic pattern of As biotransformation genes?

\section{Results}

\section{Physiochemical characteristics of sediment samples}

A total of 70 samples were collected from 14 estuarine sediments across 4 provinces in Southeastern China, ranging from $21.6^{\circ}$ to $30.4^{\circ} \mathrm{N}$ and $108.3^{\circ}$ to $121.7^{\circ} \mathrm{E}$ (Supporting Information Fig. S1 and Table S2). Tm varied from $20.4^{\circ} \mathrm{C}$ to $30.2^{\circ} \mathrm{C}$, and $\mathrm{pH}$ varied from 6.5 to 8.1 . The $\mathrm{C} / \mathrm{N}$ ratio varied from 9.2 to 26.8. Total As concentrations in these samples were below the Class I Sediment Quality Criteria (20 mg kg ${ }^{-1}$, National Standard of P.R. China, 2002), and half of the samples were below the low interim sediment quality value for As concentrations in wetlands (8.2 $\mathrm{mg} \mathrm{kg}^{-1}$, Hongkong ISQVs). Ferric iron (Fe(III)) was dominant in total $\mathrm{Fe}$, which ranged from 15.1 to $27.2 \mathrm{~g}$ $\mathrm{kg}^{-1}$ and accounted for $94 \%-99 \%$ of the total Fe. Significant positive correlations for $\mathrm{Tm}$ and $\mathrm{pH}$ values with geographic indicators (latitude and longitude) were detected by Spearman's rank-order correlation analysis $(P<0.05)$. Total $\mathrm{C}$ concentrations significantly correlated with total $\mathrm{N}$ concentrations $(P<0.05)$ (Supporting Information Table S3).

\section{Abundance and biodiversity of genes involved in as biotransformation}

Wide range of gene abundance levels and diversities of aio $A$, arr $A, \operatorname{ars} C$ and $\operatorname{ars} M$ genes were detected in all 70 samples (Fig. 1 and Supporting Information Table S4). The relative abundance levels of ars $C$ genes were significantly $(P<0.01)$ higher than the other three genes (Supporting Information Fig. S2), ranging from $2.3 \times 10^{-3}$ to $2.9 \times$ $10^{-1}$. Unexpectedly, ars $C$ genes were even more 

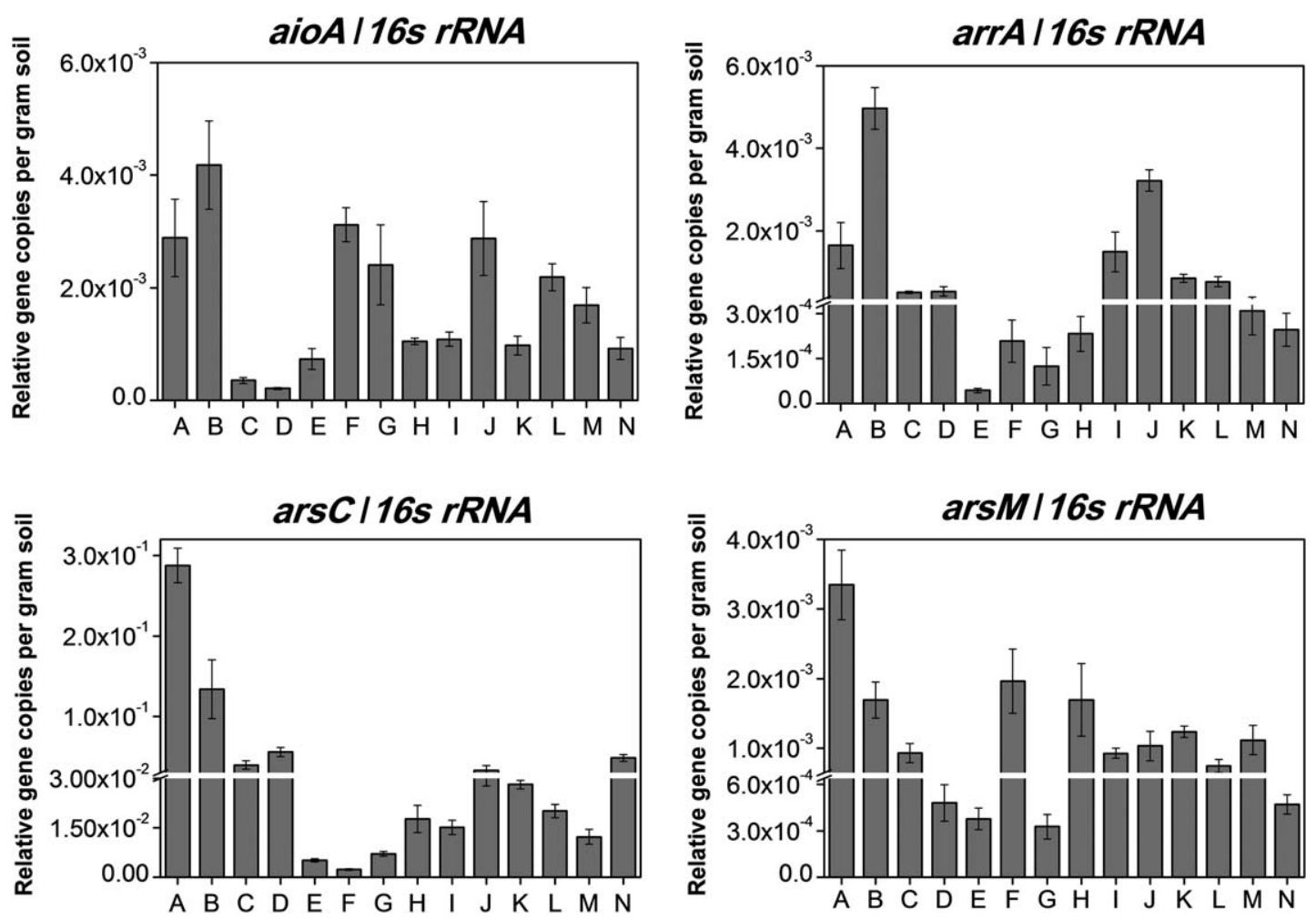

Fig. 1. Detected levels of aio $A$, arrA, ars $C$ and ars $M$ genes normalized to copy numbers of $16 \mathrm{~S}$ rRNA genes present in 14 estuarine sediments collected across Southeastern China. The absolute gene copy numbers of aioA, arrA, ars $C$ and ars $M$ were normalized to that of ambient 16S rRNA genes, in order to minimize variances caused by different background bacterial abundances, extraction and analytical efficiencies. Error bars indicate the standard deviation of five replicates.

abundant than in As-contaminated paddy soils (Jia et al., 2014; Zhang et al., 2015). The relative gene abundance levels of the aioA, arrA and ars $M$ genes varied from $2.1 \times$ $10^{-4}$ to $4.2 \times 10^{-3}, 4.4 \times 10^{-5}$ to $5.0 \times 10^{-3}$ and $3.3 \times$ $10^{-4}$ to $3.4 \times 10^{-3}$, respectively, one magnitude lower than (aio $A$ gene) or comparable to (arrA and ars $M$ genes) those detected in paddy soil.

Functional microbial communities involved in As biotransformation were examined by terminal restriction fragment length polymorphism (T-RFLP) analysis (Supporting Information Fig. S3). The aioA genes were dominated by terminal restriction fragments (T-RFs) 80,88 and $130 \mathrm{bp}$, while the ars $C$ genes were dominated by $\mathrm{T}-$ RFs 53 and $146 \mathrm{bp}$, and the ars $M$ genes were dominated by T-RFs 59 and 83 bp. For arrA, T-RF 34 bp was the dominant T-RF in almost all samples except sample $E$ (FJHTX), which was dominated by T-RF 58 bp. Shannon diversity indices based on the T-RFLP profiles indicated that the alpha-diversities of the aio $A$ genes were significantly higher than the others $(P<0.001)$ (Supporting Information Fig. S4), but all As biotransformation genes showed comparable alpha-diversities to those detected in As contaminant paddy soils (Jia et al., 2014; Zhang et al., 2015).
Geographic distribution patterns of genes involved in as biotransformation

Principle coordinates analysis (PCOA) of functional microbial communities revealed four clusters for aioA, arrA and ars $C$ genes based on the T-RFLP profiles (Fig. 2). However, for the arsM genes, no clear clustering of the functional microbial communities was observed based on the T-RFLP profiles (Supporting Information Fig. S5), possibly due to the low resolution of the T-RFLP technique for ars $M$ genes. In addition, the biases associated with the widely used digestion enzyme (Taq) in T-RFLP analysis for ars $M$ genes should also be considered since the choice of different digestion enzymes could generate highly variable species richness and diversity indices (Zhang et al., 2008). Therefore, MiSeq sequencing analysis of arsM genes was performed to generate a high-resolution profile. The rarefaction curves of Shannon index approached the plateau phase, indicating adequate sequencing depth (Wang et al., 2012) for arsM in these samples (Supporting Information Fig. S6), and microbial community richness and diversity across sampling sites were revealed (Supporting Information Table S5). Functional microbial communities based on ars $M$ MiSeq sequencing data were 

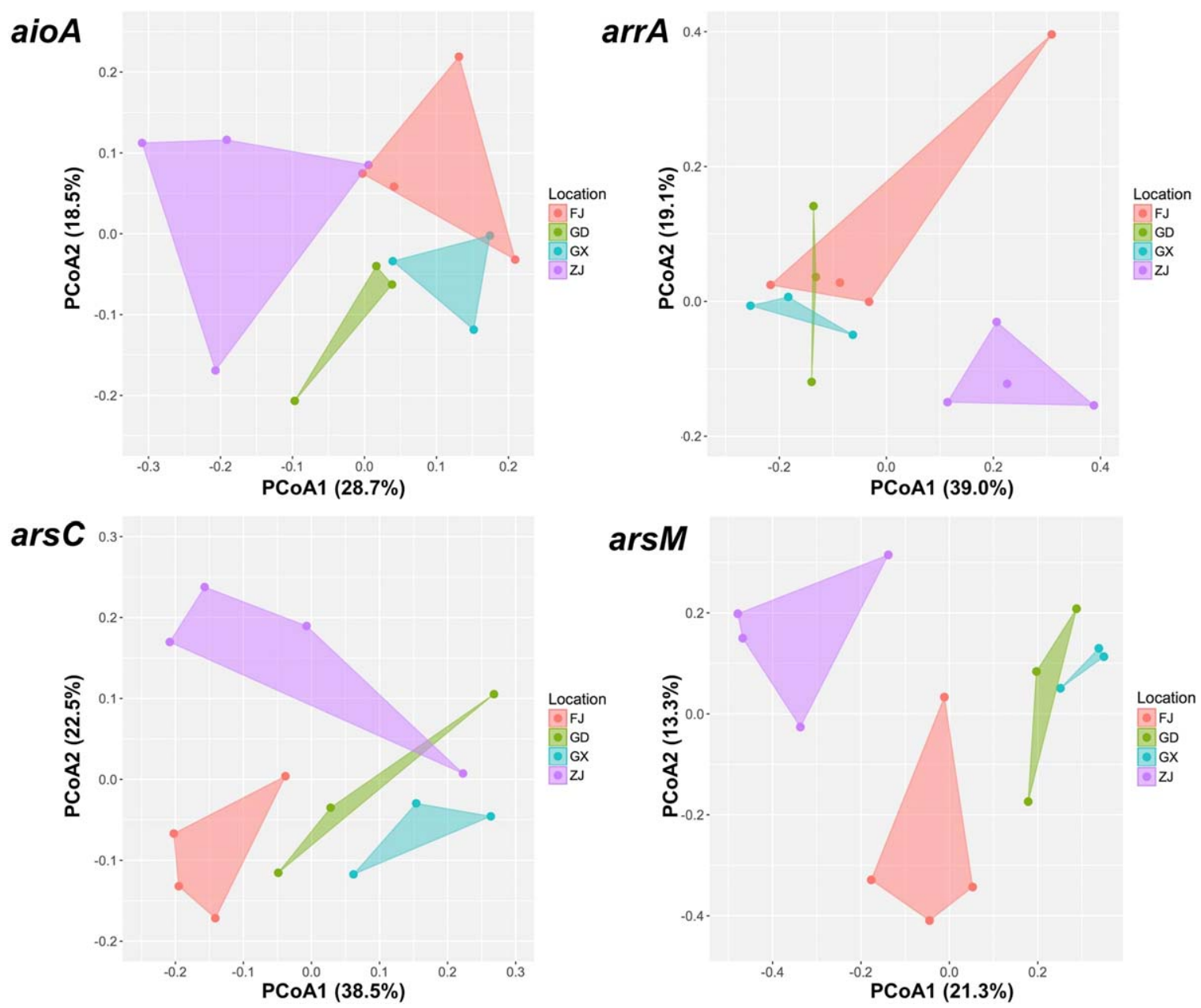

Fig. 2. Principal coordinate analysis (PCoA) of Bray-Curtis distances generated from T-RFLP profiles for aioA, arrA and ars $C$ genes, and MiSeq sequencing data for ars $M$ genes. Proportion of variance explained by each principal coordinate axis is denoted in the corresponding axis label. Data sets coloured by different colours indicate samples from different locations (red: Fujian province, green: Guangdong province, blue: Guangxi province, purple: Zhejiang province). [Color figure can be viewed at wileyonlinelibrary.com]

clustered into four groups (Fig. 2), corresponding to the geographic patterns for microbes involved in As redox.

The Adonis dissimilarity test corroborated that the geographic distribution patterns imposed changes in microbial community compositions involved in As biotransformation. Samples located at $28.0^{\circ}$ to $30.4^{\circ} \mathrm{N}$ and $120.6^{\circ}$ to $121.7^{\circ} \mathrm{E}$ (Zhejiang province), $24.4^{\circ}$ to $26.6^{\circ} \mathrm{N}$ and $117.9^{\circ}$ to $119.6^{\circ}$ E (Fujian province), $22.8^{\circ}$ to $23.4^{\circ} \mathrm{N}$ and $113.6^{\circ}$ to $116.2^{\circ}$ E (Guangdong province) and $21.6^{\circ}$ to $21.9^{\circ} \mathrm{N}$ and $108.3^{\circ}$ to $109.1^{\circ} \mathrm{E}$ (Guangxi province) showed significant $(P<0.01$ or 0.05$)$ dissimilarities in the functional microbial community compositions for aio $A$, arr $A$ and $\operatorname{ars} C$ genes based on T-RFLP profiles and for ars $M$ genes based on MiSeq sequencing data (Supporting Information Table S6). Microbes involved in As(III) methylation displayed a significant, negative distance-decay curve (Pearson correlation value $=0.512, P<0.001)$ (Fig. 3 ), corresponding to the significant decay of the microbial community similarity over geographic distance. The distance-decay relationship was also significant for the 16S rRNA genes (Pearson correlation value $=0.371, P<0.001)$, but it was weaker than that of arsM (Supporting Information Fig. S7).

\section{Taxonomic profiling of microbes involved in As(III) methylation and their co-occurrence patterns}

Microbes involved in As(III) methylation were mostly assigned to Proteobacteria (Alpha-, Beta-, Delta- and Gamma-), Methanomicrobia, Actinobacteria, Clostridia, Planctomycetia, Halobacteria, Thermomicrobia and Opitutae (Fig. 4). However, $6 \%$ to $33 \%$ (average 17\%) of the total microbes were unclassified, and $28 \%$ to $58 \%$ (average $42 \%$ ) of the total microbes were identified as uncultured bacteria from the environmental samples. The top 100 abundant As(III)-methylating microbes at the family level are classified in a heatmap (Supporting 


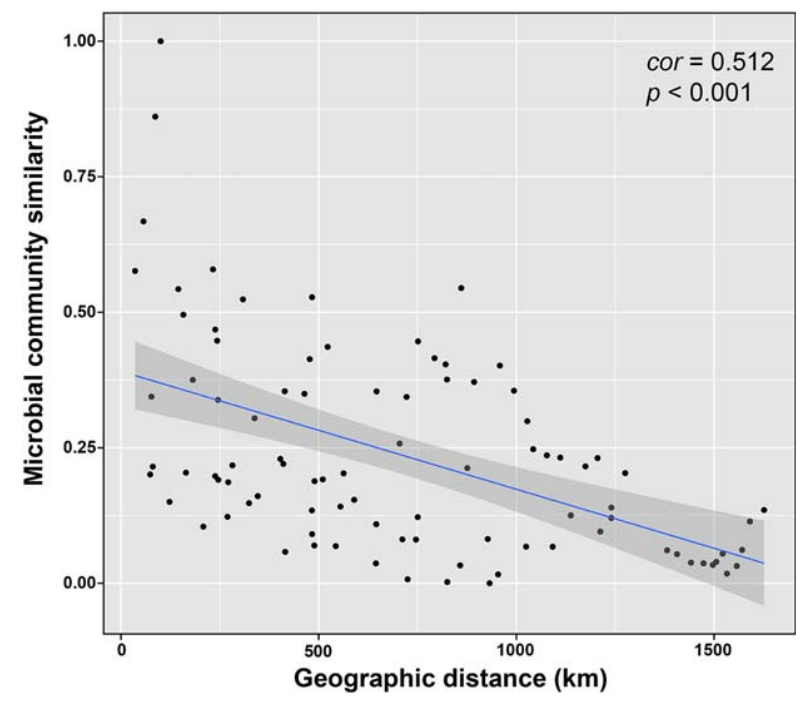

Fig. 3. Distance-decay curve for microbial community similarity based on arsM genes. Bray-Curtis distances for microbial community and geographic distances among samples were calculated and plotted to generate Pearson correlation values (cor). The shadow indicates the $95 \%$ confidence intervals. [Color figure can be viewed at wileyonlinelibrary.com]

Information Fig. S8), and their abundance levels in total microbes involved in As(III) methylation were calculated (Supporting Information Table S7). Bradyrhizobiaceae and Methanosaetaceae were the most abundant microbes involved in As(III) methylation, followed by Clostridiales XVIII, Planctomycetaceae, Halobacteriaceae, Nocardioidaceae, Sphaerobacteraceae, Hyphomicrobiaceae, Hydrogenophilaceae and Polyangiaceae. Eukaryota including Euteleostomi and Chlorellaceae were also detected, but with relatively low abundance $(0.3 \%$ and $0.04 \%$ respectively).

The co-occurrence patterns of microbes involved in As(III) methylation are shown by network inference (Fig. 5). The resulting network consisted of 49 nodes (OTUs) and 129 edges with a node connectivity of 2.63. The clustering coefficient (Newman, 2003) was 0.22 , and the modularity index was 0.50 (values $>0.4$ ), suggesting that the network has a modular structure (Newman, 2006). At the family level, microbes represented by the 49 OTUs in the network included Bradyrhizobiaceae, Clostridiales XVIII, Opitutaceae, Sphaerobacteraceae, Hyphomicrobiaceae, Planctomycetaceae, Polyangiaceae, Nocardioidaceae, Halobacteriaceae, Desulfobacteraceae, Methanosaetaceae, and some uncultured and unclassified microbes. Further analysis of the relative abundance levels of these microbes in random occurrence in all samples and in the co-occurrence network (Supporting Information Fig. S9) showed that Clostridiales XVIII, Opitutaceae, Sphaerobacteraceae, Hyphomicrobiaceae, Polyangiaceae and Desulfobacteraceae were more abundant in co-occurrence network (14.9\%, $11.9 \%$, $9.9 \%, 6.9 \%, 5.0 \%$ and $2.0 \%$, respectively) than random occurrence in the total microbes involved in As(III) methylation $(10.6 \%, 1.8 \%, 4.8 \%, 3.8 \%, 3.0 \%$ and $1.3 \%$ respectively). This finding suggests that these microbes tend to co-occur more than what would be expected by chance. Bradyrhizobiaceae was the most abundant microbe both in the total $(36.7 \%)$ microbes and in the cooccurrence network (31.7\%). The second most abundant microbe, Methanosaetaceae, in total microbes (18.9\%) was the least abundant in the co-occurrence network $(2.0 \%)$.

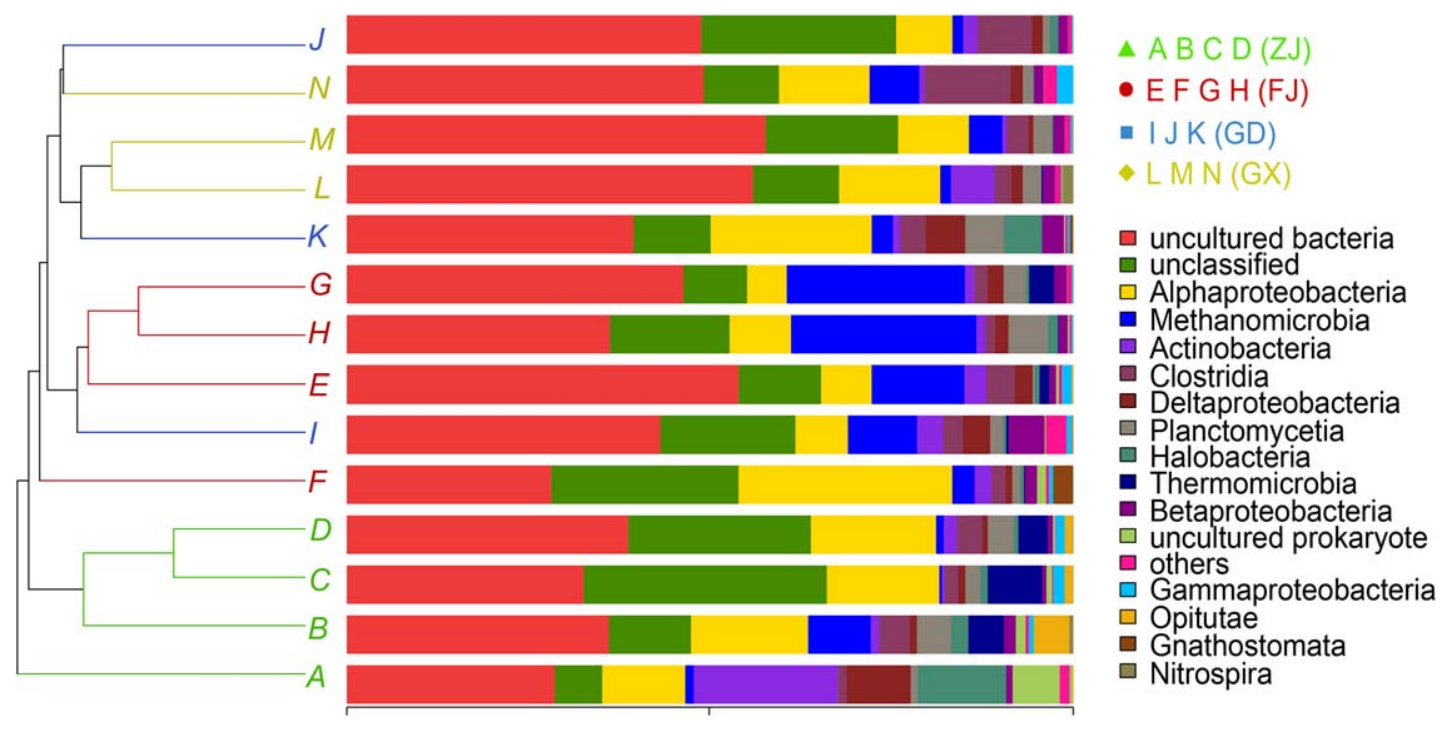

Fig. 4. Class-level diversity chart for microbial communities involved in As(III) methylation in different sediment samples arranged by hierarchical cluster analysis of Bray-Curtis distances. [Color figure can be viewed at wileyonlinelibrary.com] 


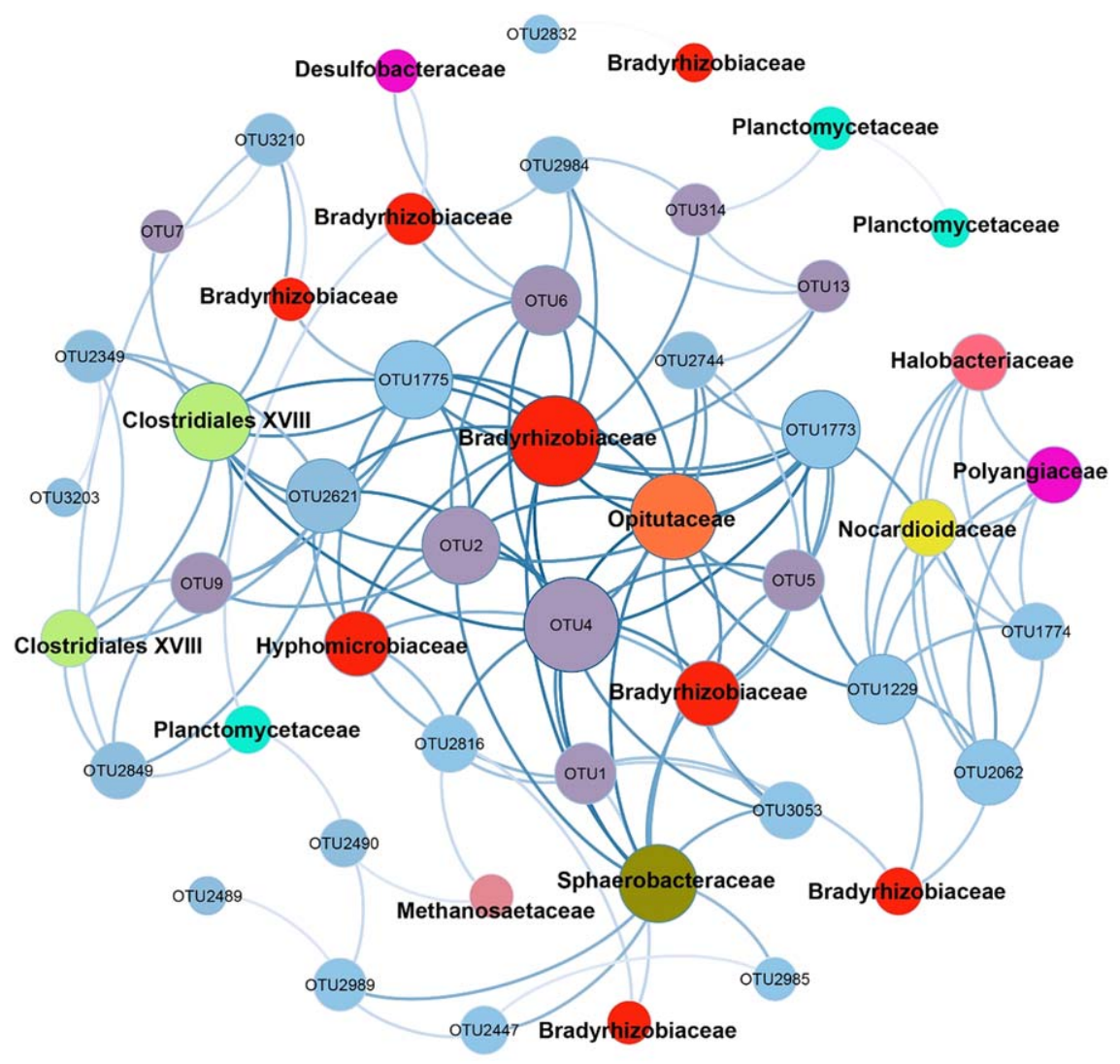

unclassified

Opitutae

Actinobacteria
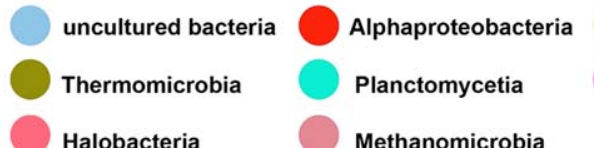

Methanomicrobia
Fig. 5. Network of co-occurring $97 \%$ cutoff OTUs based on correlation analysis. A connection stands for a strong (correlation coefficient $>0.6)$ and significant $(P$ value $<0.01$ ) correlation. The 52 OTUs in the network were forward selected with abundance $>0.3 \%$ to total OTUs based on arsM genes. The size of each node is proportional to the number of connections (that is, degree). [Color figure can be viewed at wileyonlinelibrary.com]
Environmental factors affecting gene abundance levels and microbial biogeographic patterns involved in as biotransformation

Redundancy analysis (RDA) of the environmental factors affecting As biotransformation gene abundances and microbial biogeographic patterns involved in As redox (aioA, arrA and ars $C$ genes based on T-RFLP profiles) revealed that significant models had a confidence level of $P<0.01$ (Supporting Information Fig. S10). Latitude, Tm and total $\mathrm{N}$ and $\mathrm{C} / \mathrm{N}$ ratio significantly correlated with the variation in the relative As gene abundance levels, and the first axis accounted for the majority of the total variance (96.4\%). For As(III)-oxidizing microbes (aioA), latitude, $\mathrm{pH}$ and $\mathrm{Tm}$ were the significant environmental factors affecting the microbial community compositions, and the first and second axis explained $26.1 \%$ and $11.9 \%$ of the total variances respectively. For microbes involved in $\mathrm{As}(\mathrm{V})$ respiratory reduction (arrA), latitude, $\mathrm{pH}, \mathrm{Fe}(\mathrm{III})$ and $\mathrm{Tm}$ were significantly linked to the variances in the microbial community compositions with $34.8 \%$ and $14.3 \%$ explained by the first and second axis. The variances in microbial
Clostridia

Deltaproteobacteria

community compositions involved in $\mathrm{As}(\mathrm{V})$ detoxification reduction $(\operatorname{ars} C)$ were significantly shaped by longitude, latitude, $\mathrm{pH}, \mathrm{C} / \mathrm{N}$ ratio and $\mathrm{Tm}$. The first and second axis explained $31.3 \%$ and $10.2 \%$ of total variances.

The soil properties were further separated to soil attributes, including $\mathrm{pH}, \mathrm{C} / \mathrm{N}$ ratio, total $\mathrm{C}, \mathrm{N}, \mathrm{Fe}, \mathrm{As}$ and $\mathrm{Fe}(\mathrm{III})$ concentrations, and geographic factors including latitude, longitude and Tm. Tm was included in geographic factors because $\mathrm{Tm}$ and latitude are not independent and will nearly always co-vary in costal environments, which was also demonstrated by the significant Spearman rank-order correlation (rho $=-0.705$ ) of $\mathrm{Tm}$ and latitude across all sites (Supporting Information Table S3). Canonical correspondence analysis (CCA) based on arsM MiSeq sequencing data revealed that latitude, longitude, $\mathrm{C} / \mathrm{N}$ ratio, $\mathrm{pH}, \mathrm{Tm}$, total $\mathrm{N}$ and $\mathrm{Fe}(\mathrm{III})$ concentrations significantly explained the variation in the microbial community compositions involved in $\mathrm{As}(\mathrm{III})$ methylation $(P=0.003)$, and the first and second axis accounted for $14.0 \%$ and $12.6 \%$ of the total variance respectively (Fig. $6 \mathrm{~A}$ ). Variance partition analysis revealed that a total of $62.9 \%$ of the 
A

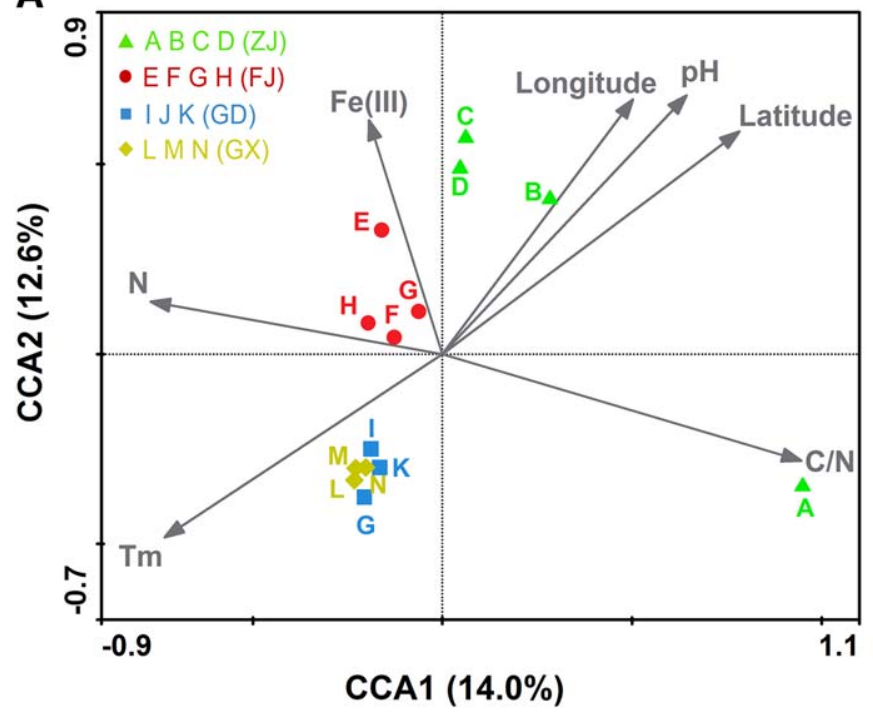

B

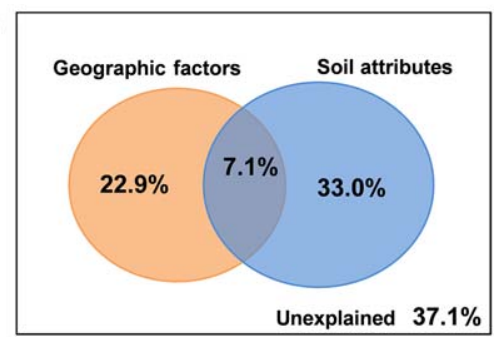

C

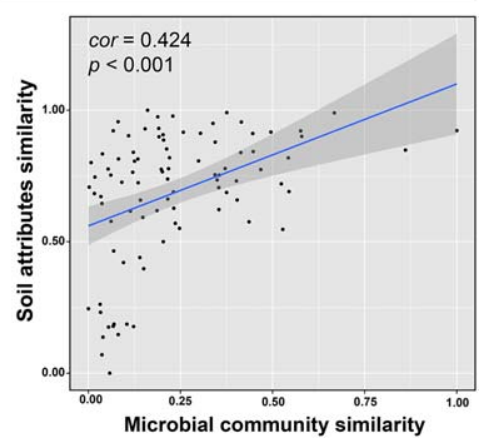

Fig. 6. A. Canonical correspondence analyses (CCA) of arsM microbial communities based on Miseq sequencing profiles with environmental factors (arrows). Environmental explanatory variables were forward selected based on the significance test performed by Monte Carlo permutation test $(P<0.05)$. Symbols in different colours indicate samples from different provinces (green: Zhejiang province, red: Fujian province, blue: Guangdong province, yellow: Guangxi province). The values of CCA1 and 2 are percentages that the corresponding axis can explain.

B. Variation partitioning analysis that partitions relative influence of environmental factors on microbial community structure. Environmental factors are divided into groups of geographic factors (Latitude, Longitude, Tm) and soil attributes $(\mathrm{C} / \mathrm{N}, \mathrm{pH}, \mathrm{N}, \mathrm{Fe}(\mathrm{III}))$. The overlap represents the joint effect of the two groups.

C. The correlations between microbial community and soil attributes. Bray-Curtis distances for microbial community and Euclid distances for soil attributes among samples were calculated and plotted to generate Pearson correlation values (cor). The shadow indicates the $95 \%$ confidence intervals. [Color figure can be viewed at wileyonlinelibrary.com]

community variations were explained by these selected variables (Fig. 6B). Among them, soil attributes $(\mathrm{C} / \mathrm{N}, \mathrm{pH}$, total $\mathrm{N}, \mathrm{Fe}(\mathrm{III}))$ independently accounted for $33.0 \%$ of the total variances, and showed strong (Pearson correlation value $=0.424)$ and significant correlations $(P<0.001)$ with microbial compositions based on the ars $M$ genes in the similarity test (Fig. 6C). Geographic factors (latitude, longitude and $\mathrm{Tm}$ ) independently explained $22.9 \%$ of the total variances. Soil attributes and geographic factors interactively explained $7.1 \%$ of the total variances, which possibly resulted from the significant correlation $(P<0.01$ or 0.05$)$ of $\mathrm{pH}$ values with the geographic factors (Supporting Information Table S3). A total of $37.1 \%$ of the total variances remained unexplained by these environmental factors.

The environmental factors revealed in CCA and RDA were verified by Mantel tests on the basis of their significant correlation with microbial gene abundance levels and community compositions involved in As biotransformation (Table 1 and Supporting Information Table S8). The heterogeneity of local environmental factors was demonstrated using a significant distance-decay relationship (Pearson correlation value $=0.263, P<0.01)$ of soil attributes $(\mathrm{pH}$,

Table 1. Correlation between microbial community involved in As(III) methylation (arsM) based on MiSeq sequencing profiles and environmental factors as shown by Mantel tests.

\begin{tabular}{|c|c|c|c|}
\hline Environmental factor (unit) & $r$ value & Environmental factor (unit) & $r$ value \\
\hline Latitude & $0.670^{\star * c}$ & $N\left(\mathrm{~g} \mathrm{~kg}^{-1}\right)$ & $0.338^{*}$ \\
\hline Longitude & $0.373^{\star \star}$ & $\mathrm{C} / \mathrm{N}$ ratio & $0.346^{\star}$ \\
\hline $\operatorname{Tm}\left({ }^{\circ} \mathrm{C}\right)$ & $0.502^{\star *}$ & $\mathrm{Fe}\left(\mathrm{g} \mathrm{kg}^{-1}\right)$ & $0.220^{\star}$ \\
\hline $\mathrm{pH}$ & $0.582^{\star \star \star}$ & $\mathrm{Fe}(\mathrm{III})\left(\mathrm{g} \mathrm{kg}^{-1}\right)$ & $0.231^{*}$ \\
\hline $\mathrm{C}\left(\mathrm{g} \mathrm{kg}^{-1}\right)$ & 0.079 & As $\left(\mathrm{mg} \mathrm{kg}^{-1}\right)$ & 0.056 \\
\hline Geographic factors ${ }^{a}$ & $0.627^{\star \star \star}$ & Soil attributes ${ }^{b}$ & $0.565^{\star \star *}$ \\
\hline
\end{tabular}

a. Geographic factors: Latitude, Longitude, Tm (Temperature).

b. Soil attributes: $\mathrm{pH}, \mathrm{N}, \mathrm{C} / \mathrm{N}$ ratio, $\mathrm{Fe}(\mathrm{III})$.

c. Significant differences $(P<0.05)$ are indicated in bold. ${ }^{*} P<0.05,{ }^{* *} P<0.01,{ }^{* *} P<0.001$. 
Table 2. AIC values for 6 rank abundance distribution models based on MiSeq sequencing profiles of arsM genes. Lowest AIC value for each sample represents the best fit model.

\begin{tabular}{|c|c|c|c|c|c|c|}
\hline \multirow[b]{2}{*}{ Sample ID } & \multicolumn{5}{|c|}{ Niche } & \multirow{2}{*}{$\frac{\text { Neutra }}{\text { ZSM }}$} \\
\hline & Broken-stick & Pre-Emption & Log-Normal & Zipf & Zipf-Mandelbrot & \\
\hline$A$ & 37559 & 17183 & $1652^{*}$ & 3181 & 1835 & 8689 \\
\hline B & 24814 & 15259 & $2438^{*}$ & 3325 & 2596 & 5975 \\
\hline C & 21814 & 11248 & $1613^{*}$ & 2449 & 1672 & 4633 \\
\hline $\mathrm{D}$ & 47922 & 29270 & 5035 & 5293 & $4045^{\star}$ & 6887 \\
\hline$E$ & 16338 & 12379 & $2889^{*}$ & 4665 & 4132 & 7356 \\
\hline $\mathrm{F}$ & 38792 & 27486 & 4053 & 3660 & $3173^{*}$ & 8031 \\
\hline $\mathrm{G}$ & 29261 & 26289 & 6275 & $4988^{\star}$ & 4990 & 11013 \\
\hline $\mathrm{H}$ & 27753 & 24502 & 5299 & $3521^{*}$ & 3523 & 9506 \\
\hline I & 15133 & 11617 & $3311^{*}$ & 4049 & 3399 & 9947 \\
\hline $\mathrm{J}$ & 26316 & 18941 & 4957 & 5822 & $3536^{*}$ & 10410 \\
\hline $\mathrm{K}$ & 17848 & 9541 & 3344 & 5447 & $2376^{\star}$ & 7898 \\
\hline $\mathrm{L}$ & 13483 & 10440 & 3833 & 5543 & $3725^{\star}$ & 11063 \\
\hline $\mathrm{M}$ & 34303 & 27466 & 4857 & 3912 & $3751^{*}$ & 11242 \\
\hline $\mathrm{N}$ & 22960 & 18636 & 3569 & $3146^{*}$ & 3148 & 8774 \\
\hline
\end{tabular}

*The best fit model with lowest AIC value.

total $\mathrm{N}, \mathrm{C} / \mathrm{N}$ ratio and $\mathrm{Fe}(\mathrm{III})$ (Supporting Information Fig. S11).

\section{A niche-based theory explained the assembly of microbial communities}

The comparison of different rank abundance distribution models based on AIC values showed that the data based on ars $M$ genes in different sediments best fit either with the Log-normal, Zipf or Zipf-Mandelbrot models (Table 2), indicating that the niche-based mechanism best explained the composition and structure of the microbial communities involved in As(III) methylation. Similarly, the data based on the 16S rRNA genes in different sediments were also fit with the Zipf-Mandelbrot model, suggesting that the nichebased mechanism primarily shaped the taxonomic microbial community (Supporting Information Table S10).

\section{Discussion}

Prevalence of microbes involved in as biotransformation distributed in estuarine sediments

The ubiquitous presence of microbes capable of $\mathrm{As}(\mathrm{III})$ respiratory oxidation, $\mathrm{As}(\mathrm{V})$ respiratory reduction, $\mathrm{As}(\mathrm{V})$ detoxification reduction and $\mathrm{As}$ (III) methylation in all estuarine sediments was demonstrated in this study (Fig. 1). Among them, ars $C$ had the highest abundance among the four As biotransformation genes (Supporting Information Fig. S2); strikingly, it was even more abundant than those detected in As contaminant paddy soils (Jia et al., 2014; Zhang et al., 2015). The Great Oxidation of the Earth resulted in more $\mathrm{As}(\mathrm{V})$ in the environment. To detoxify $\mathrm{As}(\mathrm{V})$, microbial ArsC enzymes evolved to reduce $\mathrm{As}(\mathrm{V})$ to As(III) and subsequently pump it out (Mukhopadhyay et al., 2002). The high abundance levels of $\mathrm{As}(\mathrm{V})$ reduction-related genes (ars) have also been observed in low As environments (Cai et al., 2013; Xiao et al., 2016), corroborating the prevalence of microbes capable of $\mathrm{As}(\mathrm{V})$ detoxification via reduction in these estuarine sediments. Additionally, estuaries are adjacent to the marine environment, in which $A s(V)$ accumulation by microbes is elevated via the phosphate uptake system due to the low phosphate levels in the marine environment (Sanders, 1980; Takahashi et al., 1990; Zhang et al., 2013a). Although it is not experimentally validated in this study, it is likely that microbes in these environments may acquire more abundant $\operatorname{ars} C$ genes to detoxify the highly accumulated $A s$ in their cytoplasm.

The abundance of As(III) respiratory oxidizing microbes $(a i o A)$ and respiratory $\mathrm{As}(\mathrm{V})$ reducing microbes (arrA) were lower in sediments (Fig. 1) but still comparable to those detected in As-contaminated paddy soils (Jia et al., 2014; Zhang et al., 2015). The low As levels in these estuarine sediments could explain the lower relative abundance, suggesting that As respiration may not be an important process to either detoxify As or couple it to ATP production (Ahmann, 1994; Slyemi and Bonnefoy, 2012) due to the lack of available As. Nevertheless, the highest Shannon diversity detected in aioA genes (Supporting Information Fig. S4) indicated that As(III)-oxidizing microbes are still very diverse. In addition, the previously identified dominant T-RFs for aioA genes (Jia et al., 2014; Zhang et al., 2015) were also detected in this study (Supporting Information Fig. S3), demonstrating the presence of dominant $\mathrm{As}$ (III)-oxidizing microbes in these estuarine sediments.

The surface of ancient earth might have had more abundant As than it has today (Oremland et al., 2009), and As 
metabolic pathways subsequently evolved as important processes for early cellular life to either couple the element to energy production or mitigate its toxicity (Lebrun et al., 2003; Oremland and Stolz, 2003; Silver and Phung, 2005; Zhu et al., 2014). Therefore, it is likely that the prevalence of As biotransformation genes in the environment resulted from this history.

In-depth investigation of microbes involved in As(III) methylation distributed in estuarine sediments

The ars $M$ genes responsible for As(III) methylation are considered to be involved in an important As detoxification mechanism (Zhu et al., 2014). The comparable abundant ars $M$ genes detected in this study showed great potential for As(III) methylation in the estuarine environment. However, the present study of ars $M$ genes is still limited since ars $M$ gene references in the $\mathrm{NCBI}$ database are insufficient, thus resulting in the high percentage of unclassified ars $M$ gene sequences (17\%) or those identified as uncultured bacteria from environmental samples (42\%) (Supporting Information Table S7). Among the classified microbes involved in As(III) methylation were microbes belonging to Planctomycetes, Chloroflexi, Verrucomicrobia and Eukaryotes (Fig. 4), along with those belonging to Proteobacteria, Actinobacteria, Firmicutes and Euryarchaeota, which were previously identified in paddy soils (Jia et al., 2013; Zhao et al., 2013; Zhang et al., 2015). To the best of our knowledge, arsM genes assigned to Eukaryotes have never been observed in environmental samples, possibly due to the limited number of sequences based on clone library analysis (Jia et al., 2013; Zhang et al., 2015), the limited arsM sequences on the GeoChip (Zhao et al., 2013), or the reduced sensitivity of metagenomics for identifying functional genes (Cai et al., 2013; Luo et al., 2014; Xiao et al., 2016). However, Eukaryotes are prevalent in estuarine environments, and their importance in $\mathrm{As}$ (III) methylation warrants further investigation, especially considering the confirmed As(III) methylation capacity of Eukaryotes, such as Tetrahymena thermophila (Yin et al., 2011a) and Ostreococcus tauri (Zhang et al., 2013b).

Close associations of Alphaproteobacteria with Opitutae and Thermomicrobia, and Actinobacteria with Deltaproteobacteria and Halobacteria were observed for microbes involved in As(III) methylation (Fig. 5), possibly contributing to the relative higher abundance of these microbes detected in sediments from Zhejiang province (Sample A, B, C and D, Fig. 4). While previous studies showed that co-occurrence patterns mostly occurred in closely related phylogenetic taxa (Barberan et al., 2012), our study demonstrated the relatively high co-occurrence patterns of microbes from different taxa, indicating a different niche for functional microbial communities. Nevertheless, the relatively high fraction of unclassified OTUs in the network might be an important reason why we hardly detected cooccurrence patterns derived from closely related phylogenetic taxa. The co-occurrence patterns could be potential indicators of competitive interactions, non-overlapping niches or historical effects (Horner-Devine et al., 2007). Clostridiales XVIII, Opitutaceae, Sphaerobacteraceae, Hyphomicrobiaceae, Polyangiaceae and Desulfobacteraceae tend to co-occur more than expected by chance (Supporting Information Fig. S9), suggesting niche adaptation of these microbes involved in As(III) methylation. However, Methanosaetaceae tended to co-occur less than expected by chance, suggesting their independence and restricted habitat preference in sediments from the Fujian province (Sample E, F, G and H, Supporting Information Table S7).

\section{Processes and factors driving microbial biogeography associated with as biotransformation}

Our results showed that the mechanisms for shaping both taxonomic microbial communities (based on the 16S rRNA gene amplicons) and the functional microbial communities involved in As(III) methylation (based on the arsM gene amplicons) were consistent with deterministic processes (Table 2 and Supporting Information Table S10). Intriguingly, the variations in functional microbial community compositions involved in As biotransformation were unrelated to the As levels (Fig. 6A and Supporting Information Fig. S10). Our results collectively suggest that at large spatial scales, other environmental factors, rather than As levels, are crucial in shaping the functional microbial communities involved in As(III) methylation. This conclusion is reasonable because As levels in all samples were relatively low and similar; such correlation would be expected in As-rich environment and with various in situ bioavailable As.

The significant correlations between soil attributes (with high spatial heterogeneity, Supporting Information Fig. $\mathrm{S} 11$ ), and the microbial community based on ars $M$ genes (Fig. 6C) corroborated the concept that the assembly based on niche-based mechanisms is mainly determined by the niche requirements and local habitat conditions (Chase and Myers, 2011). The reason for latitude as the main factor influencing both abundance and diversity of As biotransformation genes is possibly due to that factors showing strong linkages with As biotransformation microbes such as $\mathrm{Tm}, \mathrm{pH}$ and $\mathrm{N}$ concentration, which exhibit significant correlation with latitude (Supporting Information Table S3). The significant effect of $\mathrm{pH}$ on microbial community compositions involved in As biotransformation could be due to the fact that soil $\mathrm{pH}$ was important for determining the ionized and associated forms of As in As biotransformation (Vahter, 2002), and As 
biotransformation processes in paddy soils were sensitive to $\mathrm{pH}$ variation (Stolz and Oremland, 1999). In addition, we found that $\mathrm{Fe}(\mathrm{III})$ in estuarine sediments was linked to microbial biogeographic patterns associated with As biotransformation, especially for microbes involved in $\mathrm{As}(\mathrm{V})$ respiratory reduction and As(III) methylation (Fig. 6 and Supporting Information Fig. S10). It is well known that iron oxyhydroxides could sequester As(V) (Liu et al., 2006), reducing As bioavailability to microbes, and possibly contribute to the less abundance of arrA genes detected in samples from Fujian province (E, F, G and H) (Fig. 1) in which $\mathrm{Fe}(\mathrm{III})$ concentrations were higher compared with the other samples (Supporting Information Table S2). Since microbe-mediated redox processes are strongly related to the microbial accessibility of $\mathrm{C}$ and $\mathrm{N}$ (KoegelKnabner et al., 2010), it is reasonable to expect that $\mathrm{C} / \mathrm{N}$ ratio would also be significant environmental factor affecting the abundance of As biotransformation microbes. Moreover, the effect of $\mathrm{N}$ concentration on microbial community compositions involved in As(III) methylation has also been demonstrated previously (Zhang et al., 2015).

\section{Microbial biogeography: Taxonomic and functional communities}

The functional similarity (Pearson correlation value $=0.512$ for ars $M$ genes) showed a higher distance-decay rate than the phylogenetic similarity (Pearson correlation value $=0.371$ for $16 \mathrm{~S}$ rRNA genes), suggesting a quicker turnover of the functional genes than that found in microbial species. The higher fraction of variances (33.0\%) in the microbial community involved in As(III) methylation was explained independently by soil attributes, including $\mathrm{pH}$, total $\mathrm{N}, \mathrm{C} / \mathrm{N}$ ratio and $\mathrm{Fe}(\mathrm{III})$, rather than by geographic factors (Fig. 6B). In contrast, among the soil attributes, only $\mathrm{pH}$ was significantly correlated with the taxonomic microbial community (Supporting Information Table S9). Considering the substitutability of microorganisms, microbial taxa distributions have been suggested to be of little value in predicting environmental changes (Green et al., 2008). Rather, a function-based approach might be essential for understanding the importance of the specific habitats of microbial functional traits in the environment (Burke et al., 2011). It has been shown that metabolic flexibility can be a major predictor for the spatial distribution within microbial communities (Carbonero et al., 2014), representing a distinct microbial ecology principle that differs from macro-ecology. We reinforce this viewpoint in our study by showing that the functional composition of microbes is correlated to soil geochemical parameters. Therefore, in-depth metagenomics and metatranscriptomics profiling will provide a large amount of data that is amenable for exploration to recognize bio-indicators of environmental conditions.
Implications of functional biogeography to as biotransformation genes evolution and as biogeochemical cycle

This study is the first to demonstrate the wide presence of As biotransformation genes (aio $A$, arr $A$, ars $C$ and $\operatorname{ars} M$ ) in an environment with a low As level, which is possibly a legacy of active As metabolism in early life (Sforna et al., 2014). While previous studies have demonstrated the diversity of As biotransformation microbes (Oremland and Stolz, 2003), we further disclosed the biogeographic microbial patterns associated with As biotransformation at a large spatial scale. Since the low As level in these sediments was not strong enough to impose selection on As biotransformation genes, heterogeneity of local soil attributes may lead to variable selection for taxonomic microbial communities (Dini-Andreote et al., 2015), which may become more important in determining functional microbial biogeographic patterns. Compared with microbial taxonomy, environmental selection for functional genes seems to be less complicated, rendering functional microbial communities as an ideal model to study the important role of deterministic processes in shaping microbial biogeographic patterns. Moreover, our study revealed the ubiquity of the abundant and diverse microbes involved in As redox reactions and methylation in estuarine sediments at a large spatial scale. While As redox reactions determine the dynamic of inorganic As species in estuarine wetlands (Islam et al., 2004; Bhattacharya et al., 2007), As(III) methylation can facilitate the emission of As from sediments to the atmosphere because the end products of this process are volatile As species (Chilvers and Peterson, 1987; Mestrot et al., 2011). Therefore, it is not surprising that high methylating capacity was found in sediments where As concentrations were low. Considering the prevalence of microbes capable of As biotransformation, the high capacity of As biotransformation in environments and the large, global area of estuarine wetlands, these microbes are critical to the global As biogeochemical cycle.

\section{Experimental procedures}

\section{Sites description and soil chemistry}

A total of 70 sediment samples from depths of $0-15 \mathrm{~cm}$ were collected in 2013 from 14 estuarine wetlands (five replicates for each sample) distributed in 4 provinces in Southeastern China (Supporting Information Fig. S1). These sites included, the regions of Zhejiang-Qiantangjiang (A: ZJ-QTJ), ZhejiangYongjiang (B: ZJ-YJ), Zhejiang-Jiaojiang (C: ZJ-JJ), ZhejiangOujiang (D: ZJ-OJ), Fujian-Huotongxi (E: FJ-HTX), FujianMinjiang (F: FJ-MJ), Fujian-Jinjiang (G: FJ-JJ), FujianJiulongjiang (H: FJ-JLJ), Guangdong-Hanjiang (I: GD-HJ), Guangdong-Longjiang (J: GD-LJ), Guangdong-Zhujiang (K: GD-ZJ), Guangxi-Nanliujiang (L: GX-NLJ), Guangxi-Qinjiang (M: GX-QJ) and Guangxi-Fangchengjiang (N: GX-FCJ). Sediments samples were placed in sterile plastic bags and 
transported to the laboratory on ice for DNA extraction and soil chemistry analysis. Soil properties, including $\mathrm{pH}$, the concentration of total carbon $(\mathrm{C})$, nitrogen $(\mathrm{N}), \mathrm{As}$, iron $(\mathrm{Fe})$ and ferric iron $(\mathrm{Fe}(\mathrm{III}))$, and the $\mathrm{C} / \mathrm{N}$ ratio were analysed. Details of the measurement methods are shown in the Supporting information.

DNA extraction and PCR-based analysis of aioA, arrA, ars $C$ and arsM

DNA was extracted from $0.5 \mathrm{~g}$ of the samples using the FastDNA® SPIN kit for soil (MP Biomedicals) according to the manufacturer's instructions and was checked for quality by agarose gel electrophoresis and ultraviolet-absorbance (ND1000, Nanodrop, Thermo Fisher Scientific, Inc.). DNA yields of the 70 sediment samples were between 4 to $116 \mathrm{ng}$ $\mu \mathrm{L}^{-1}$ with most of them arranged from 20 to $60 \mathrm{ng} \mu \mathrm{L}^{-1}$. The ratio of absorbance at $260 \mathrm{~nm}$ and $280 \mathrm{~nm}$ was around 1.8. Quantitative real-time polymerase chain reaction (qPCR) performed on an iQTM5 Thermocycler (Bio-Rad, USA) was conducted to investigate the aioA, arrA, ars $C$, arsM and $16 \mathrm{~S}$ rRNA gene abundance levels. The aio $A$, arrA, ars $C$ and ars $M$ genes were normalized by dividing the absolute gene copy numbers by that of $16 \mathrm{~S}$ rRNA. Terminal restriction fragment length polymorphism (T-RFLP) analysis was conducted to investigate aio $A$, arrA, ars $C$ and $\operatorname{ars} M$ gene diversities. For each sampling site, the relative abundance levels of terminal restriction fragments (T-RFs) were calculated by normalizing the peak areas of each individual T-RF to the peak areas of the total T-RFs. T-RFs that occurred in at least five sampling replicates and with percentages $>2 \%$ were listed. Details are shown in the Supporting information.

\section{High-throughput MiSeq sequencing of the arsM and $16 S$ rRNA genes}

Total genomic DNA was PCR amplified using the primers arsMF1 and arsMR2 (Jia et al., 2013) with barcodes, targeting a conserved $\sim 347$ bp region of the arsM genes. PCR conditions were: $95^{\circ} \mathrm{C}$ for $5 \mathrm{~min}, 35$ amplification cycles of $95^{\circ} \mathrm{C}$ for $30 \mathrm{~s}, 62^{\circ} \mathrm{C}$ for $35 \mathrm{~s}, 72^{\circ} \mathrm{C}$ for $1 \mathrm{~min}$, and a final single extension cycle of $72^{\circ} \mathrm{C}$ for $1 \mathrm{~min}$. Three replicates PCRs were performed for each DNA sample, and amplicons generated from each set of the three reactions were subsequently pooled and quantified using PicoGreen. Negative controls and no template controls were included for DNA extraction and PCR amplification. Finally, the five barcoded amplicons for each sample site were pooled in equal concentrations for sequencing. The amplicon pool was purified using the MoBio UltraClean PCR Clean-up kit and was sequenced on the Illumina MiSeq sequencing platform (MiSeq Control Software 2.0.5 and Real-Time Analysis software 1.16.18) at Majorbio Bio-pharm Technology Company in Shanghai, China. The 14 pools of amplicons were sequenced using the 300PE protocol, which generated a total of 393980 raw read pairs. The sequence read pre-processing was performed in FLASH (Magoč and Salzberg, 2011), yielding a total of 316975 trimmed sequences that varied from 17371 to 28468 per sample. The final average sequence length after the barcode, heterogeneity spacer and primer removal was $324 \mathrm{bp}$.
Processing of the ars $M$ gene's sequence reads was performed using QIIME (version 1.6.0) (Caporaso et al., 2010), including additional quality trimming and demultiplexing. Operational taxonomic units (OTUs) were calculated at the $97 \%$ sequence identity level and resulted in 3211 OTUs. The most abundant sequence from each OTU was selected as a representative sequence for that OTU. Taxonomy identification was performed using the Basic Local Alignment Search Tool (BLAST) for each representative sequence against the arsM gene sequences from the $\mathrm{NCBI}$ database. Shannon and Simpson diversity, abundance-based coverage estimators (ACE) and Chao richness for each sample of the sites were calculated in R 3.1.2 with the fossil package (Vavrek, 2012). Details of the 16S rRNA gene sequencing process were shown in the Supporting information. Gene sequences data of arsM and 16S rRNA genes were submitted to NCBI under the accession number: KP678028 - KP681238 for arsM genes and SRP076214 for 16S rRNA genes.

\section{Data analyses}

Principle coordinates analysis (PCoA) and the Adonis dissimilarity test based on T-RFLP or MiSeq sequencing data were conducted to examine the distribution patterns of microbial community compositions involved in As biotransformation. Monte Carlo permutation was used to test the statistical significance. The distance-decay analysis was applied for the arsM genes to investigate the declining microbial community similarity with geographic distance, and tested by Pearson correlation coefficient. The distance-decay analysis based on the 16S rRNA genes was also performed to compare the distance-decay rates for functional and taxonomic microbial communities, and details are shown in the Supporting information. The similarity and Mantel tests were performed to evaluate the linkages between functional or taxonomic microbial groups and environmental factors, followed by calculation of the Pearson correlation coefficient. In these analyses, the Bray-Curtis distance for microbial communities and the Euclid distance for environmental factors were used to calculate the distance between samples. Redundancy analysis (RDA) or Canonical correspondence analysis (CCA) were chosen according to the result of the detrended correspondence analysis (DCA). Environmental explanatory variables were forward selected based on the significance test performed by the Monte Carlo permutation test $(P<0.05)$. The variation partitioning analysis resulted in three geographic factors (latitude, longitude and sediment temperature (Tm)) and four soil attributes $(\mathrm{C} / \mathrm{N}, \mathrm{pH}, \mathrm{N}, \mathrm{Fe}(\mathrm{III}))$. Spearman's rank-order correlation was used to test the correlations between the environmental factors. This non-parametric method was used because these data did not follow a normal distribution. All of the analyses were performed in R 3.1.2 with vegan (Oksanen et al., 2013) and ggplot2 (Wickham, 2009) packages.

To explore the co-occurrence patterns in soil microbial communities involved in As(III) methylation, network analysis was conducted by calculating the inferred correlations between the forward selected 52 OTUs (with abundance $>0.3 \%$ to total OTUs) using Sparse Correlations for Compositional data (SparCC) (Friedman and Alm, 2012; Weiss et al., 2016). If the correlation coefficient was $>0.6$ and statistically significant $(P$ value $<0.01$ ), a valid co-occurrence event was identified as a 
robust correlation. The nodes in the reconstructed network represent the OTUs at $97 \%$ identity, and the edges correspond to a strong and significant correlation between nodes. The network was explored and visualized with the interactive platform Gephi (Bastian et al., 2009).

The rank abundance distribution was examined to test whether niche-based or neutral models best explain the assembly of the microbial community involved in As(III) methylation. The niche-based theory predicts that the rank abundance distribution would fit the broken stick, pre-emption, log-normal, Zipf and Zipf-Mandelbrot models (Motomura, 1932; MacArthur, 1957; McGill et al., 2007). The neutral theory assumes that the rank abundance distribution would be consistent with ZSM (Hubbell, 2001). A measure of the relative quality of a statistical model, i.e., the Akaike Information Criterion (AIC) value, was used to compare which model provided the best fit to the empirical data (Dumbrell et al., 2010; Mendes et al., 2014). The best fit model was indicated by the lowest AIC value. The analyses of the broken stick, preemption, log-normal, Zipf and Zipf-Mandelbrot models were performed in R 3.1.2 with the vegan package (Oksanen et al., 2013), and the ZSM model was performed using Tetame (Jabot et al., 2008).

\section{Acknowledgements}

This study is financially supported by Strategic Priority Research Program of Chinese Academy of Sciences (XDB15020402) and National Natural Science Foundation of China (41430858, 41090282). We gratefully thank ChemWorx for English-language editing. Xubiao Luo is also appreciated for his editorial assistance. We declare that there is no conflict of interest for this study.

\section{References}

Aguilar, M., Fiore-Donno, A.-M., Lado, C., and Cavalier-Smith, T. (2014) Using environmental niche models to test the 'everything is everywhere' hypothesis for Badhamia. ISME J 8: 737-745.

Ahmann, D. (1994) Microbe grows by reducing arsenic. Nature 371: 750.

Barberan, A., Bates, S.T., Casamayor, E.O., and Fierer, N. (2012) Using network analysis to explore co-occurrence patterns in soil microbial communities. ISME J 6: 343-351.

Bastian, M., Heymann, S., and Jacomy, M. (2009) Gephi: an open source software for exploring and manipulating networks. ICWSM 8: 361-362.

Bhattacharjee, H., and Rosen, B.P. (2007) Arsenic metabolism in prokaryotic and eukaryotic microbes. In Molecular Microbiology of Heavy Metals. Nies, D.H., Silver, S. (eds). Heidelberg, Germany: Springer Press, pp. 371-406.

Bhattacharya, P., Welch, A.H., Stollenwerk, K.G., McLaughlin, M.J., Bundschuh, J., and Panaullah, G. (2007) Arsenic in the environment: Biology and Chemistry. Sci Total Environ 379: 109-120.

Burke, C., Steinberg, P., Rusch, D., Kjelleberg, S., and Thomas, T. (2011) Bacterial community assembly based on functional genes rather than species. Proc Natl Acad Sci USA 108: 14288-14293.
Cai, L., Yu, K., Yang, Y., Chen, B.-W., Li, X.-D., and Zhang, T. (2013) Metagenomic exploration reveals high levels of microbial arsenic metabolism genes in activated sludge and coastal sediments. Appl Microbiol Biotechnol 97: 9579-9588.

Caporaso, J.G., Kuczynski, J., Stombaugh, J., Bittinger, K., Bushman, F.D., Costello, E.K., et al. (2010) QIIME allows analysis of high-throughput community sequencing data. Nat Methods 7: 335-336.

Carbonero, F., Oakley, B.B., and Purdy, K.J. (2014) Metabolic flexibility as a major predictor of spatial distribution in microbial communities. PLoS One 9: e85105.

Chase, J.M., and Myers, J.A. (2011) Disentangling the importance of ecological niches from stochastic processes across scales. Philos Trans $R$ Soc Lond B: Biol Sci 366: 23512363.

Chilvers, D., and Peterson, P. (1987) Global cycling of arsenic. In Lead, Mercury, Cadmium and Arsenic in the Environment. Hutchinson, T.C., Meema, K.M. (eds). New York, USA: Wiley.

Dini-Andreote, F., Stegen, J.C., Van Elsas, J.D., and Salles, J.F. (2015) Disentangling mechanisms that mediate the balance between stochastic and deterministic processes in microbial succession. Proc Natl Acad Sci USA 112: 1326-1332.

Dumbrell, A.J., Nelson, M., Helgason, T., Dytham, C., and Fitter, A.H. (2010) Relative roles of niche and neutral processes in structuring a soil microbial community. ISME J 4: 337-345.

Duval, S., Ducluzeau, A.-L., Nitschke, W., and SchoeppCothenet, B. (2008) Enzyme phylogenies as markers for the oxidation state of the environment: the case of respiratory arsenate reductase and related enzymes. BMC Evol Biol 8: 1-13.

Escudero, L.V., Casamayor, E.O., Chong, G., Pedrós-Alió, C., and Demergasso, C. (2013) Distribution of microbial arsenic reduction, oxidation and extrusion genes along a wide range of environmental arsenic concentrations. PLoS One 8: e78890.

Friedman, J., and Alm, E.J. (2012) Inferring correlation networks from genomic survey data. PLoS Comput Biol 8: $1-11$.

Green, J.L., Bohannan, B.J., and Whitaker, R.J. (2008) Microbial biogeography: from taxonomy to traits. Science $\mathbf{3 2 0}$ 1039-1043.

Hambright, K.D., Beyer, J.E., Easton, J.D., Zamor, R.M., Easton, A.C., and Hallidayschult, T.C. (2015) The niche of an invasive marine microbe in a subtropical freshwater impoundment. ISME J 9: 256-264.

Horner-Devine, M.C., Silver, J.M., Leibold, M.A., Bohannan, B.J., Colwell, R.K., Fuhrman, J.A., et al. (2007) A comparison of taxon co-occurrence patterns for macro-and microorganisms. Ecology 88: 1345-1353.

Hubbell, S.P. (2001) The Unified Neutral Theory of Biodiversity and Biogeography (MPB-32). New Jersey, USA: Princeton University Press.

Islam, F.S., Gault, A.G., Boothman, C., Polya, D.A., Charnock, J.M., Chatterjee, D., et al. (2004) Role of metal-reducing bacteria in arsenic release from Bengal delta sediments. Nature 430: 68-71.

Jabot, F., Etienne, R.S., and Chave, J. (2008) Reconciling neutral community models and environmental filtering: theory and an empirical test. OIKOS 117: 1308-1320. 
Jia, Y., Huang, H., Zhong, M., Wang, F.-H., Zhang, L.-M., and Zhu, Y.-G. (2013) Microbial arsenic methylation in soil and rice rhizosphere. Environ Sci Technol 47: 3141-3148.

Jia, Y., Huang, H., Chen, Z., and Zhu, Y.-G. (2014) Arsenic uptake by rice is influenced by microbe-mediated arsenic redox changes in the rhizosphere. Environ Sci Technol 48: 1001-1007.

Koegel-Knabner, I., Amelung, W., Cao, Z., Fiedler, S., Frenzel, P., Jahn, R., et al. (2010) Biogeochemistry of paddy soils. Geoderma 157: 1-14.

Kulp, T.R. (2014) Early earth: Arsenic and primordial life. Nat Geosci 7: 785-786.

Lebrun, E., Brugna, M., Baymann, F., Muller, D., Lièvremont, D., Lett, M.-C., et al. (2003) Arsenite oxidase, an ancient bioenergetic enzyme. Mol Biol Evol 20: 686-693.

Liu, W.J., Zhu, Y.-G., Hu, Y., Williams, P.N., Gault, A.G., Meharg, A.A., et al. (2006) Arsenic sequestration in iron plaque, its accumulation and speciation in mature rice plants (Oryza sativa L.). Environ Sci Technol 40: 5730-5736.

Luo, J., Bai, Y., Liang, J., and Qu, J. (2014) Metagenomic approach reveals variation of microbes with arsenic and antimony metabolism genes from highly contaminated soil. PLoS One 9:1-9.

MacArthur, R.H. (1957) On the relative abundance of bird species. Proc Natl Acad Sci USA 43: 293-295.

Magoč, T., and Salzberg, S.L. (2011) FLASH: fast length adjustment of short reads to improve genome assemblies. Bioinformatics 27: 2957-2963.

Maguffin, S.C., Kirk, M.F., Daigle, A.R., Hinkle, S.R., and Jin, Q. (2015) Substantial contribution of biomethylation to aquifer arsenic cycling. Nat Geosci 8: 290-293.

Malasarn, D., Saltikov, W., Campbell, K.M., Santini, J.M., Hering, J.G., and Newman, D.K. (2004) arrA is a reliable marker for $\mathrm{As}(\mathrm{V})$ respiration. Science 306: 455-455.

Martiny, J.B.H., Bohannan, B.J., Brown, J.H., Colwell, R.K., Fuhrman, J.A., Green, J.L., et al. (2006) Microbial biogeography: putting microorganisms on the map. Nat Rev Microbiol 4: 102-112.

Martiny, J.B., Eisen, J.A., Penn, K., Allison, S.D., and Horner-Devine, M.C. (2011) Drivers of bacterial $\beta$-diversity depend on spatial scale. Proc Natl Acad Sci USA 108: 7850-7854.

McGill, B.J., Etienne, R.S., Gray, J.S., Alonso, D., Anderson, M.J., Benecha, H.K., et al. (2007) Species abundance distributions: moving beyond single prediction theories to integration within an ecological framework. Ecol Lett 10: 995-1015.

Mendes, L.W., Kuramae, E.E., Navarrete, A.A., Van Veen, J.A., and Tsai, S.M. (2014) Taxonomical and functional microbial community selection in soybean rhizosphere. ISME J 8: 1577-1587.

Mestrot, A., Feldmann, J., Krupp, E.M., Hossain, M.S., Roman-Ross, G., and Meharg, A.A. (2011) Field fluxes and speciation of arsines emanating from soils. Environ Sci Technol 45: 1798-1804.

Motomura, I. (1932) On the statistical treatment of communities. Zool Mag 44: 379-383.

Mukhopadhyay, R., Rosen, B.P., Phung, L.T., and Silver, S. (2002) Microbial arsenic: from geocycles to genes and enzymes. FEMS Microbiol Rev 26: 311-325.

Newman, M.E. (2003) The structure and function of complex networks. SIAM Rev 45: 167-256.
Newman, M.E. (2006) Modularity and community structure in networks. Proc Natl Acad Sci USA 103: 8577-8582.

Oksanen, J., Blanchet, F.G., Kindt, R., Legendre, P., Minchin, P.R., O'Hara, R., et al. (2013) Package "vegan": community ecology. R package version 2.0-7. The Comprehensive $R$ Network (CRAN).

Oremland, R.S., and Stolz, J.F. (2003) The ecology of arsenic. Science 300: 939-944.

Oremland, R.S., Saltikov, C.W., Wolfe-Simon, F., and Stolz, J.F. (2009) Arsenic in the evolution of earth and extraterrestrial ecosystems. Geomicrobiol J 26: 522-536.

Qin, J., Rosen, B.P., Zhang, Y., Wang, G., Franke, S., and Rensing, C. (2006) Arsenic detoxification and evolution of trimethylarsine gas by a microbial arsenite S-adenosylmethionine methyltransferase. Proc Natl Acad Sci USA 103: 20752080.

Sanders, J.G. (1980) Arsenic cycling in marine systems. Mar Environ Res 3: 257-266.

Sforna, M.C., Philippot, P., Somogyi, A., Van Zuilen, M.A., Medjoubi, K., Schoepp-Cothenet, B., et al. (2014) Evidence for arsenic metabolism and cycling by microorganisms 2.7 billion years ago. Nat Geosci 7: 811-815.

Silver, S., and Phung, L.T. (2005) Genes and enzymes involved in bacterial oxidation and reduction of inorganic arsenic. Appl Environ Microbiol 71: 599-608.

Slyemi, D., and Bonnefoy, V. (2012) How prokaryotes deal with arsenic. Environ Microbiol Rep 4: 571-586.

Stolz, J.F., and Oremland, R.S. (1999) Bacterial respiration of arsenic and selenium. FEMS Microbiol Rev 23: 615-627.

Takahashi, A., Kawakami, H., Bada, A., Okonogi, Y., and Matsuto, S. (1990) Effects of phosphate on arsenate inhibition in a marine cyanobacterium, Phormidium sp. Appl Organomet Chem 4: 269-279.

Vahter, M. (2002) Mechanisms of arsenic biotransformation. Toxicology 181: 211-217.

Vavrek, M. (2012) Package "fossil": Palaeoecological and palaeogeographical analysis tools. $\mathrm{R}$ package version 0.3.7. The Comprehensive R Network (CRAN).

Wang, Y., Sheng, H.-F., He, Y., Wu, J.-Y., Jiang, Y.-X., Tam, N.F.-Y., et al. (2012) Comparison of the levels of bacterial diversity in freshwater, intertidal wetland, and marine sediments by using millions of illumina tags. Appl Environ Microbiol 78: 8264-8271.

Wang, P.-P., Sun, G.-X., and Zhu, Y.-G. (2014) Identification and characterization of arsenite methyltransferase from an archaeon, Methanosarcina acetivorans C2A. Environ Sci Technol 48: 12706-12713.

Weiss, S., Van Treuren, W., Lozupone, C., Faust, K., Friedman, J., Deng, Y., et al. (2016) Correlation detection strategies in microbial data sets vary widely in sensitivity and precision. ISME J 10: 1669-1681.

Wickham, H. (2009) ggplot2: elegant graphics for data analysis, 3rd ed. New York, NY: Springer Press.

Xiao, K.-Q., Li, L.-G., Ma, L.-P., Zhang, S.-Y., Bao, P., Zhang, T., et al. (2016) Metagenomic analysis revealed highly diverse microbial arsenic metabolism genes in paddy soils with low-arsenic contents. Environ Pollut 211: 1-8.

Yin, X.-X., Zhang, Y.-Y., Yang, J., and Zhu, Y.-G. (2011a) Rapid biotransformation of arsenic by a model protozoan Tetrahymena thermophila. Environ Pollut 159: 837-840. 
Yin, X.-X., Chen, J., Qin, J., Sun, G.-X., Rosen, B., and Zhu, Y.-G. (2011b) Biotransformation and volatilization of arsenic by three photosynthetic cyanobacteria. Plant Physiol 156: 1631-1638.

Zedler, J.B., and Kercher, S. (2005) Wetland resources: status, trends, ecosystem services, and restorability. Annu Rev Environ Resour 30: 39-74.

Zhang, R., Thiyagarajan, V., and Qian, P.-Y. (2008) Evaluation of terminal-restriction fragment length polymorphism analysis in contrasting marine environments. FEMS Microbiol Ecol 65: 169-178.

Zhang, S.-Y., Rensing, C., and Zhu, Y.-G. (2013a) Cyanobacteria-mediated arsenic redox dynamics is regulated by phosphate in aquatic environments. Environ Sci Technol 48: 994-1000.

Zhang, S.-Y., Sun, G.-X., Yin, X.-X., Rensing, C., and Zhu, Y.G. (2013b) Biomethylation and volatilization of arsenic by the marine microalgae Ostreococcus tauri. Chemosphere 93: 47-53.

Zhang, S.-Y., Zhao, F.-J., Sun, G.-X., Su, J.-Q., Yang, X.-R., $\mathrm{Li}, \mathrm{H}$., et al. (2015) Diversity and abundance of arsenic biotransformation genes in paddy soils from Southern China. Environ Sci Technol 49: 4138-4146.

Zhang, S.-Y., Williams, P.N., Luo, J.M., and Zhu, Y.-G. (2017) Microbial mediated arsenic biotransformation in wetlands. Front Environ Sci Eng 11: 1-11.

Zhao, F.-J., Harris, E., Yan, J., Ma, J., Wu, L., Liu, W., et al. (2013) Arsenic methylation in soils and its relationship with microbial arsM abundance and diversity, and As speciation in rice. Environ Sci Technol 47: 7147-7154.

Zhou, J., Deng, Y., Zhang, P., Xue, K., Liang, Y., Van Nostrand, J.D., et al. (2014) Stochasticity, succession, and environmental perturbations in a fluidic ecosystem. Proc Natl Acad Sci USA 111: 836-845.

Zhu, Y.-G., Yoshinaga, M., Zhao, F.-J., and Rosen, B.P. (2014) Earth abides arsenic biotransformations. Annu Rev Earth Planet Sci 42: 443-467.

\section{Supporting information}

Additional supporting information can be found in the online version of this article at the publisher's web-site:

Fig. S1. Location of 14 estuarine sediments collected across Southeastern China. The base map used is from the National Fundamental Geographic Information System of China.

Fig. S2. Boxplot of the relative abundance of aioA, arrA, ars $C$ and ars $M$ genes. The red triangles indicate average relative abundance of each gene. One-way analysis of variance (ANOVA) was conducted to test the difference of relative gene abundance for each gene $\left({ }^{\star \star} P<0.01\right)$.

Fig. S3. Community structure of aio $A$, arrA, ars $C$ and ars $M$ genes reflected by the terminal restriction fragment length polymorphism (T-RFLP) analysis in 14 estuarine sediments collected across Southeastern China. Terminal restriction fragments (T-RFs) were digested by Taql enzyme of PCR products of aioA, arrA, ars $C$ and ars $M$ genes from sediment samples.

Fig. S4. Boxplot of shannon diversity of aioA, arrA, ars $C$ and ars $M$ genes based on T-RFLP profiles. The red triangles indicate average shannon diversity of each gene. One- way analysis of variance (ANOVA) was conducted to test the difference of average shannon diversity for each gene $\left({ }^{* * *} P<0.001,{ }^{*} P<0.05\right)$.

Fig. S5. Principal coordinate analysis (PCoA) of Bray-Curtis distances generated from arsM genes T-RFLP profiles. Proportion of variance explained by each principal coordinate axis is denoted in the corresponding axis label. Data sets colored by different colors indicate samples from different locations (red: Fujian province, green: Guangdong province, blue: Guangxi province, purple: Zhejiang province).

Fig. S6. Rarefaction curves for shannon index based on arsM MiSeq sequencing data in samples collected from 14 estuarine sediments across Southeastern China.

Fig. S7. Distance-decay curve for microbial communities based on 16S rRNA genes. Bray-Curtis distances were used for microbial community. Pearson correlation values were calculated and plotted. The shadow indicates the $95 \%$ confidence intervals.

Fig. S8. Family-level diversity and abundance of microbe involved in As(III) methylation in 14 estuarine sediments. Complete linkage clustering of samples were calculated based on the composition and relative abundance of arsM genes. Scales of completely blue, green, yellow, orange, and red indicated the abundance of $0-0.01 \%, 0.01-0.14 \%$, $0.14-2.79 \%$, and $2.79-57.66 \%$, respectively.

Fig. S9. Relative abundance of different microbial taxonomic groups (at the family level) involved in As(III) methylation. (a) number of sequences assigned with different microbial taxonomic groups in all sediment samples. (b) number of significant co-occurrent OTUs assigned with different microbial taxonomic groups (node from Figure 5).

Fig. S10. RDA correlation biplot of relative gene abundances of aio $A$, arrA, ars $C$ and ars $M$ and aio $A$, arr $A$ and ars $C$ microbial communities based on T-RFLP profiles with environmental factors (arrows). Environmental explanatory variables were forward selected based on the significance test performed by Monte Carlo permutation test $(P<0.05)$. Symbols in different colors indicate samples from different provinces (green: Zhejiang province, red: Fujian province, blue: Guangdong province, yellow: Guangxi province). The values of RDA1 and 2 are percentages that the corresponding axis can explain.

Fig. S11. Distance-decay curve for soil attributes $(\mathrm{C} / \mathrm{N}, \mathrm{pH}$, $\mathrm{N}, \mathrm{Fe}(\mathrm{III}))$ similarity. Euclid distances for soil attributes and geographic distances among samples were calculated and plotted to generate Pearson correlation values. The shadow indicates the $95 \%$ confidence intervals.

Table S1. Details of primer pairs and qPCR thermal cycling parameters for aioA, arrA, ars C, ars $M$ and 16S rRNA genes.

Table S2. The location and soil properties of the 14 estuarine sediments collected across Southeastern China.

Table S3. Spearman rank-order correlation (rho) of environmental parameters.

Table S4. Shannon and Simpson diversity for aioA, arrA, ars $C$ and ars $M$ genes detected in each sample site calculated based on T-RFLP profiles.

Table S5. Shannon and Simpson diversity, ACE and Chao richness, and identified OTUs for each sample site based on arsM MiSeq sequencing data.

Table S6. The effect of distribution pattern on microbial community compositions involved in As biotransformation as examined by the dissimilarity test of Adonis. 
Table S7. Microbes involved in As(III) methylation detected in 14 estuarine sediments at family-level, and the percentage of their members to total microbes in all samples.

Table S8. Correlation between T-RFLP data (aio $A$, arr $A$ and ars $C$ genes) and relative gene abundances (aio $A$, arr $A$, ars $C$ and ars $M$ genes) with environmental factors as shown by Mantel tests.
Table S9. Correlation between microbial community based on 16S rRNA gene MiSeq sequencing profiles and environmental factors as shown by Mantel tests.

Table S10. AIC values for 6 rank abundance distribution models based on MiSeq sequencing profiles of $16 \mathrm{~S}$ rRNA genes. Lowest AIC value for each sample represents the best fit model. 